\title{
Mass Limits to Primordial Star Formation from Protostellar Feedback
}

\author{
Jonathan C. Tan* and Christopher F. McKee \\ *Princeton University Observatory, Peyton Hall, Princeton, NJ 08544, USA. \\ ${ }^{\dagger}$ Departments of Physics \& Astronomy, University of California, Berkeley, CA 94720, USA.
}

\begin{abstract}
How massive were the first stars? This question is of fundamental importance for galaxy formation and cosmic reionization. Here we consider how protostellar feedback can limit the mass of a forming star. For this we must understand the rate at which primordial protostars accrete, how they and their feedback output evolve, and how this feedback interacts with the infalling matter. We describe the accretion rate with an "isentropic accretion" model: $\dot{m}_{*}$ is initially very large $\left(0.03 M_{\odot} \mathrm{yr}^{-1}\right.$ when $\left.m_{*}=1 M_{\odot}\right)$ and declines as $m_{*}^{-3 / 7}$. Protostellar evolution is treated with a model that tracks the total energy of the star. A key difference compared to previous studies is allowance for rotation of the infalling envelope. This leads to photospheric conditions at the star and dramatic differences in the feedback. Two feedback mechanisms are considered: HII region breakout and radiation pressure from Lyman- $\alpha$ and FUV photons. Radiation pressure appears to be the dominant mechanism for suppressing infall, becoming dynamically important around $20 M_{\odot}$.
\end{abstract}

\section{THE COLLAPSE OF PRIMORDIAL GAS CLOUDS}

Recent numerical studies have followed the gravitational collapse of perturbations from cosmological to almost stellar dimensions [1], 2]. Baryon-dominated clouds, cooled to about 200-300 K by trace amounts of molecular hydrogen, form at the centers of dark matter halos. For $n_{\mathrm{H}}>10^{4} \mathrm{~cm}^{-3}$ the cooling rate becomes independent of density, and so the dissipation of gravitational energy in the densest regions gradually raises the temperature. In the simulation of Abel et al. [1]], the gas cloud is centrally-concentrated ( $\rho \propto r^{-k_{\rho}} ; k_{\rho} \simeq 2.2$ ) and is contracting quasi-hydrostatically with infall speeds about one third of the sound speed. In addition to thermal support, the cloud is filled with a turbulent cascade of weak shocks (T. Abel, private comm.). The structure can be described by an approximately hydrostatic polytrope with $\gamma_{p}=1+1 / n=k_{P} / k_{\rho}=$ $2\left(1-1 / k_{\rho}\right)=1.1$, where $P \propto r^{-k_{P}}$. The contraction is akin to the maximally sub-sonic Hunter[3] settling solution, for which the accretion rate is a factor $\phi_{*} \simeq 2.6$ greater than the classic Shu[5] solution. This accretion rate can be expressed in terms of the entropy parameter of the polytrope, $K=P / \rho^{\gamma_{p}}$ and the collapsed mass, $M \simeq m_{*}$,

$$
\dot{m}_{*}=\frac{8 \phi_{*}}{\sqrt{ } 3}\left[\frac{\left(3-k_{\rho}\right) k_{P}^{3} K^{3}}{2(2 \pi)^{5-3 \gamma_{p}} G^{3 \gamma_{p}-1}}\right]^{\frac{1}{2\left(4-3 \gamma_{p}\right)}} M^{j} \rightarrow 0.026 K^{\prime 15 / 7}\left(\frac{M}{M_{\odot}}\right)^{-3 / 7} M_{\odot} \mathrm{yr}^{-1},
$$

where $j \equiv 3\left(1-\gamma_{p}\right) /\left(4-3 \gamma_{p}\right)$. The numerical evaluation assumes $\phi_{*}=2.6$, since $\gamma_{p}$ is not too different from one, and $K=1.88 \times 10^{12}(T / 300 \mathrm{~K}) n_{\mathrm{H}, 4}^{-0.1} \mathrm{cgs} \equiv 1.88 \times 10^{12} K^{\prime} \mathrm{cgs}$. 
We set the temperature normalization a factor $4 / 3$ higher than is seen in simulations[1], to allow for partial pressure support from sonic and isotropic turbulent motions; i.e. $T$ is an effective temperature. The small ratio of turbulent to thermal support is in marked contrast to contemporary massive star formation[6]. In primordial clouds it is the microphysics of $\mathrm{H}_{2}$ cooling that determines both the evolution (via $\gamma_{p}$ ) and normalization (via $K^{\prime}$ and $\phi_{*}$ ) of the accretion rate. Collapse assuming constant $K$ "isentropic accretion" - agrees with 1-D numerical studies[7, 8] (Fig. 1a).

The mass-averaged rotation speed is a fraction, $f_{\text {Kep }} \simeq 0.5$, of Keplerian, approximately independent of radius[1]]. Assuming angular momentum is conserved inside the

sonic point, $r_{\mathrm{sp}}$, leads to a disk size $r_{d}=f_{\mathrm{Kep}}^{2} r_{\mathrm{sp}}=3.4\left(f_{\mathrm{Kep}} / 0.5\right)^{2}\left(M / M_{\odot}\right)^{9 / 7} K^{\prime-10 / 7} \mathrm{AU}$. Matter falls onto this disk at all radii $r \leq r_{d}$, as well as directly to the star. We follow Ulrich [9] in describing the density distribution of the rotating, freely-falling envelope.

\section{PROTOSTELLAR EVOLUTION}

At densities $n_{\mathrm{H}} \sim 10^{16} \mathrm{~cm}^{-3}$ an optically thick protostellar core forms[7], bounded by an accretion shock. The size of the protostar then changes as it accretes matter and radiates energy. For spherical geometry, the high accretion rates typical of primordial star formation lead to optically thick conditions above the accretion shock[10, 11]. Accretion energy is advected, which swells the star. However, for collapse with angular momentum, a disk forms and we expect photospheric conditions over much of the stellar surface. We employ a polytropic stellar structure and extend the energy equation model of Nakano et al.[12], that includes gravitational, ionization-dissociation, and D-burning energies, to allow for optical depth in front of the accretion shock. We also allow for some fraction of disk accretion, depending on $f_{\text {Kep }}$. We model the "luminosity wave" expansion feature [10, 11] as a relaxation to a more compact $(n=3)$ state after the star is older than its Kelvin time: to conserve energy the outer radius of the star grows by a factor, which we estimate from Omukai \& Palla[11]. We set $n=1.5$ or 3 , depending on convective stability. During optically thick accretion, before the luminosity wave, we derive an effective value for $n$ by comparing to the results of Stahler et al.[10]. Figure 1b shows this case and isentropic models with $f_{\text {Kep }}=0$ and 0.5 . The two spherical models undergo similar evolution, while the rotating model forms a smaller protostar once the photosphere retreats to the stellar surface. The protostar is then supported by D core and shell burning, as in present-day star formation. The large accretion rate causes the star to join the main sequence only at relatively high masses. The photospheric temperature is much hotter than in the spherical models, leading to significant fluxes of ionizing and FUV radiation. These form the inputs for the feedback model.

\section{FEEDBACK VERSUS ACCRETION}

The lifetimes of primordial massive stars converge to about $2 \mathrm{Myr}$ [13] so eq.(1) implies an upper limit to stellar masses of $\sim 2000 M_{\odot}$. However, other feedback processes are likely to intervene before this. Once the flux of ionizing photons from the protostar 
(a) Evolution of Mass Accretion Rate

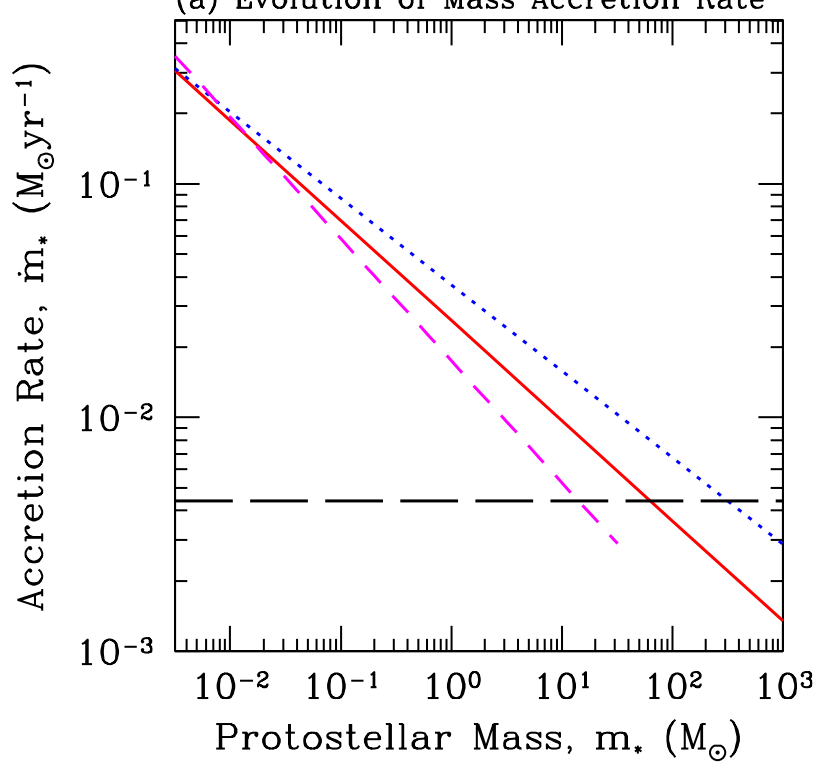

(c) HII Region Geometry near Breakout

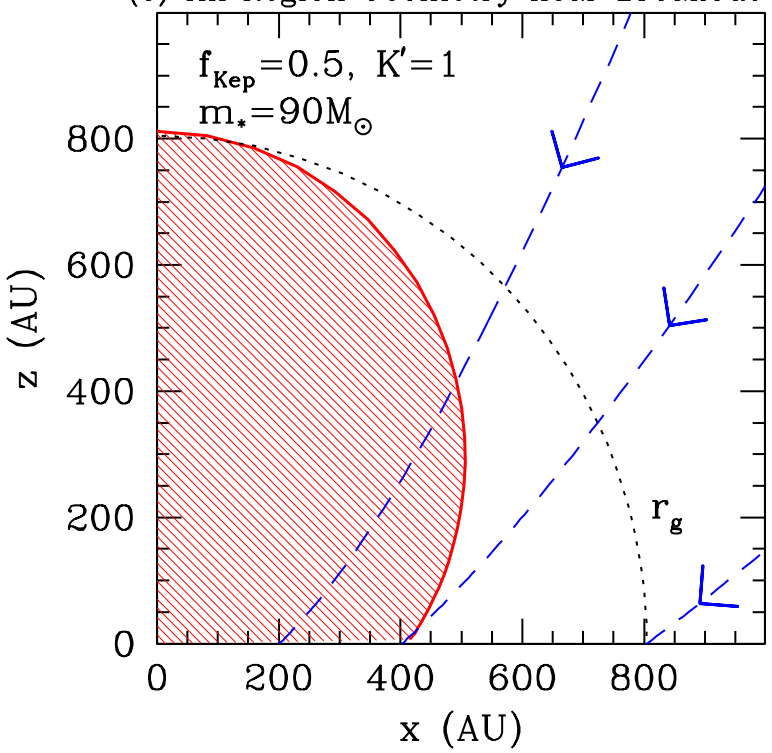

(b) Protostellar and Photospheric Radii

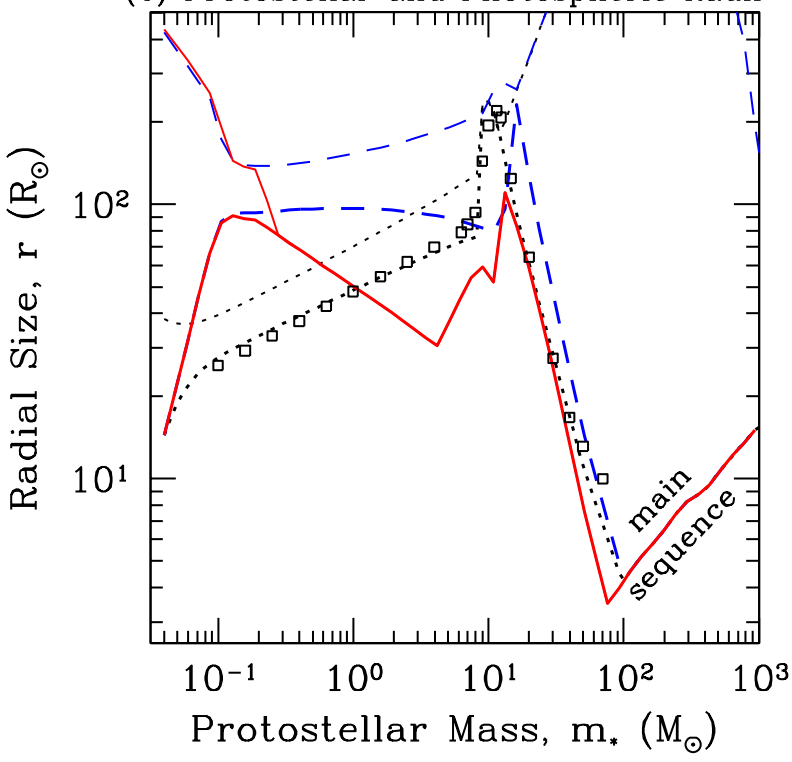

(d) Mass Scales of Feedback Processes

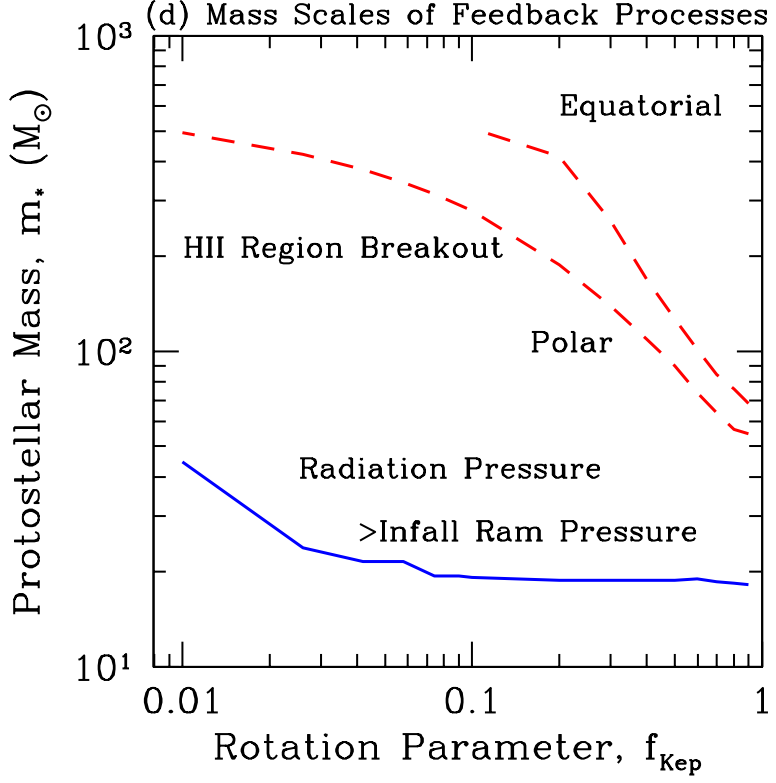

FIGURE 1. (a) Protostellar accretion rate as a function of the collapsed mass ( $\simeq m_{*}$ in these models). Solid line: fiducial isentropic accretion model $\left(K^{\prime}=1\right)$ from eq. (1); dotted[7] and dashed lines extrapolated from 1-D numerical studies; long-dashed line is $\dot{m}_{*}=4.4 \times 10^{-3} M_{\odot} \mathrm{yr}^{-1}$ used in the protostellar evolution models of Stahler et al.[10] and Omukai \& Palla[11]. (b) Evolution of protostellar radius (lower, thick lines), which is the location of the accretion shock, and photospheric radius (upper, thin lines). The spherical constant accretion rate test case (dotted) is compared to other calculations [10, 11] of $r_{*}$ (squares) for the evolution before $\mathrm{H}$ burning. Also shown are the spherical (dashed lines) and rotating $\left(f_{\mathrm{Kep}}=0.5\right.$, solid lines) isentropic accretion models. The initial condition is taken from 1-D hydrodynamical collapse simulations [8]. Note that the photospheric and protostellar radii are the same in the rotating model for $m_{*}>0.3 M_{\odot}$. (c) Geometry of the HII region (shaded) at polar breakout when $r_{\mathrm{HII}}=r_{g}$, the gravitational radius for the ionized gas sound speed. The protostar is at $(0,0)$ and the disk is in the $z=0$ plane. Dashed streamlines show infall. (d) Mass scales of feedback processes versus the rotation parameter, $f_{\text {Kep }}$. HII region breakout at the pole and just above the equator occur at masses traced by the lower and upper dashed lines, respectively. Ly- $\alpha$ and FUV radiation pressure becomes greater than twice the radial infall ram pressure (evaluated at angle $\pi / 3$ from the pole) for masses above the solid line. 
is greater than that of neutral $\mathrm{H}$ to its surface, an HII region forms. Approximating sectors as independent, we calculate the extent of the ionized region. Accretion may be suppressed if the HII region expands to distances greater than $r_{g}$, where the escape speed equals the ionized gas sound speed, $\simeq 10 \mathrm{~km} \mathrm{~s}^{-1}$. For the fiducial model this occurs at the poles when $m_{*}=90 M_{\odot}$ (Fig. 1c) and at the equator when $m_{*}=140 M_{\odot}$. In this calculation we assumed a free-fall density distribution. In reality the ionizing radiation force[14] decelerates and deflects the flow. For collapse with angular momentum, most streamlines do not come too close to the star, so the effect discussed by Omukai \& Inutsuka[14] of HII region quenching due to enhanced densities is greatly weakened. In fact, we anticipate that deflection is more important in reducing the concentration of inflowing gas near the star, so that the HII region becomes larger.

A second feedback effect is radiation pressure from Ly- $\alpha$ photons created in the HII region and FUV photons emitted by the star. These photons are trapped by the Lyman series damping wings of the neutral gas infalling towards the HII region. The energy density builds up until the escape rate, set by diffusion in frequency as well as in space[4], equals the input rate. The resulting pressure acts against the infall ram pressure. We use the results of Neufeld[15] to aid our numerical calculations. Radiation pressure becomes greater than twice the ram pressure at $m_{*} \simeq 20 M_{\odot}$ for typical $f_{\text {Kep }}$. The enhancement of radiation pressure above the optically thin limit is by a factor $\sim 10^{3}$. Infall is first reversed at the poles, which would allow photons to leak out and reduce the pressure acting in other directions. We shall examine this scenario in a future study.

\section{ACKNOWLEDGMENTS}

We thank T. Abel, V. Bromm, B. Draine, J. Goodman, D. McLaughlin, and J. Ostriker for helpful discussions. JCT is supported by a Spitzer-Cotsen fellowship from Princeton University and NASA grant NAG5-10811. The research of CFM is supported by NSF grant AST-0098365 and by a NASA grant funding the Center for Star Formation Studies.

\section{REFERENCES}

1. Abel, T., Bryan, G. L, and Norman, M. L. 2002, Science, 295, 93

2. Bromm, V., Coppi, P. S., \& Larson, R. B. 1999, Astrophys. J., 527, L5

3. Hunter, C. 1977, Astrophys. J., 218, 834

4. Adams, T. F. 1972, Astrophys. J., 174, 439

5. Shu, F. H. 1977, Astrophys. J., 214, 488

6. McKee, C. F., \& Tan, J. C. 2002, Nature, 416, 59

7. Omukai, K., \& Nishi, R. 1998, Astrophys. J., 508, 141

8. Ripamonti, E., Haardt, F., Ferrara, A., \& Colpi, M. 2002, Mon. Not. R.A.S., 334, 401

9. Ulrich, R. K. 1976, Astrophys. J., 210, 377

10. Stahler, S. W., Palla, F., \& Salpeter, E. E. 1986, Astrophys. J., 302, 590

11. Omukai, K., \& Palla, F. 2001, Astrophys. J., 561, L55

12. Nakano, T., Hasegawa, T., Morino, J.-I., \& Yamashita, T. 2000, Astrophys. J., 534, 976

13. Schaerer, D. 2002, Astron. \& Astrophys., 382, 28

14. Omukai, K., \& Inutsuka, S. 2002, Mon. Not. R.A.S., 332, 59

15. Neufeld, D. A. 1990, Astrophys. J., 350, 216 